\section{Elevada frecuencia de enfermedad tiroidea funcional en embarazadas chilenas sin antecedentes de patología tiroidea utilizando el estándar de TSH internacional}

\author{
LORENA MOSSO M. ${ }^{1}$, ALEJANDRA MARTÍNEZ G. ${ }^{\text {a }}$ \\ MARÍA PAULINA ROJAS ${ }^{2}$, PAULA MARGOZZINI ${ }^{3}$, SANDRA SOLARI ${ }^{4}$, \\ TRINIDAD LYNG ${ }^{\text {, }}$,UAN ANDRÉS ORTIZ ${ }^{5}$, JORGE CARVAJAL ${ }^{6}$
}

\section{Frequency of subclinical thyroid problems among women during the first trimester of pregnancy}

Background: Thyroid hormones play an important role in fetal neural and cognitive development. Therefore thyroid abnormalities should be detected and treated early during pregnancy. Aim: To assess the frequency and risk factors for functional thyroid disorders during the first trimester of pregnancy. Material and Methods: A blood sample was obtained from women during their first trimester of pregnancy, consulting in a prenatal care facility. Women with known thyroid diseases were excluded from the study. Thyroid stimulating hormone (TSH), total thyroxine (T4) and free thyroxine (fT4) were measured by electrochemoluminiscence. Antithyroid peroxidase antibodies (anti TPO) were measured by enzyme immunoassay. Results: Five hundred and ten women aged $25.7 \pm 6.6$ years were assessed. The frequency of clinical hypothyroidism was 0.6\%, subclinical hypothyroidism 35.3\% and clinical hyperthyroidism 1\%. Five percent of women with hypothyroidism and $3.5 \%$ of euthyroid women had positive anti TPO antibodies. There was no association between the frequency of thyroid diseases and risk factors for thyroid diseases. Conclusions: There is a high frequency of subclinical thyroid diseases among women consulting in this prenatal care clinic.

(Rev Med Chile 2012; 140: 1401-1408).

Key words: Pregnancy; Reference values; Thyroid function tests; Thyroid hormones.

\author{
'Departamento de \\ Endocrinología \\ ${ }^{2}$ Departamento de \\ Medicina Familiar. \\ ${ }^{3}$ Departamento de Salud \\ Pública. \\ ${ }^{4}$ Unidad de Laboratorio \\ Clínico. \\ ${ }^{5}$ Servicio de Ginecología y \\ Obstetricia Hospital Sótero \\ del Río. \\ ${ }^{6}$ Unidad de Medicina \\ Materno Fetal. División de \\ Obstetricia y Ginecología. \\ Facultad de Medicina. \\ Pontificia Universidad \\ Católica de Chile. \\ aResidente Endocrinología. \\ Departamento de \\ Endocrinología Facultad \\ de Medicina. Pontificia \\ Universidad Católica de \\ Chile. \\ ${ }^{\text {b} A y u d a n t e ~ A l u m n a ~}$ \\ Medicina Facultad de \\ Medicina. Pontificia \\ Universidad Católica de \\ Chile. \\ Financiamiento: Este \\ proyecto fue financiado \\ por Proyecto FONIS \\ SA10I20024 y aportes \\ de un Grant irrestricto de \\ MERCK SA. \\ Recibido el 14 de marzo de \\ 2012, aceptado el 20 de \\ junio de 2012 \\ Correspondencia a: \\ Dra. Lorena Mosso. \\ Departamento de \\ Endocrinología. Facultad \\ de Medicina. Pontificia \\ Universidad Católica de \\ Chile. Lira 85, piso 5. \\ Santiago, Chile. \\ Fono: (56-2) 354-3095 \\ Fax: (56-2) 638-5675 \\ E-mail:mosso@med.puc.cl
}

$\mathrm{E}$ n el embarazo ocurren profundos cambios fisiológicos que determinan un estado de alta demanda tiroidea. Aumenta la TBG (thyroxine-binding globulin), uniendo mayor cantidad de hormona tiroidea, haciendo necesario incrementar la producción hormonal para mantener el nivel de hormona libre normal. La gonadotrofina coriónica humana (hCG) durante el primer trimestre, posee actividad TSH símil mediante unión al receptor de TSH. Además, existe un aumento del clearance renal de yodo, aparece la deyodasa placentaria y se produce mayor metabolización hepática de T4 ${ }^{1,2}$. Estas variaciones en la función tiroidea en las embarazadas, se reflejan en los valores de referencia para las hormonas tiroideas, que son diferentes a los de la población general $^{3}$.

Se han llevado a cabo estudios en diferentes poblaciones del mundo, publicándose una variación considerable en el nivel superior de TSH para 
gestantes en las diferentes regiones. Recientemente, se han publicado consensos con los valores de normalidad de las hormonas tiroideas para cada trimestre del embarazo, las más relevantes son las guías Endocrine Society ${ }^{4}$ y la de la American Thyroid Association (ATA) ${ }^{5}$. Estas guías de consenso se basan en estudios realizados en países desarrollados, principalmente en Estados Unidos de Norteamérica y Europa.

La hormona tiroidea materna juega un rol fundamental en la progresión del embarazo y el desarrollo neurocognitivo fetal, se ha demostrado que el hipotiroidismo no tratado se asocia a elevado riesgo de parto prematuro, mortalidad perinatal y bajo peso al nacer, además de provocar alteraciones en el desarrollo neuropsicomotor del recién nacido ${ }^{6,7}$.

Si bien se ha demostrado el efecto deletéreo de la patología tiroidea funcional, no existe consenso respecto de las repercusiones perinatales o del beneficio del tratamiento de la patología tiroidea subclínica (hiper o hipotiroidismo subclínico) ${ }^{8}$. Por este motivo, se debate sobre la necesidad o el beneficio del tamizaje universal en la población de embarazadas; más aun, para apoyar una decisión correcta respecto del tamizaje universal o selectivo, es imprescindible conocer la incidencia de patología tiroidea en cada población y la capacidad predictiva del análisis según factores de riesgo.

En Chile, no contamos con estudios realizados en muestras representativas de las embarazadas que nos permita conocer la prevalencia de patología tiroidea, como tampoco se han desarrollado estudios locales que permitan conocer los rangos de normalidad de las pruebas de laboratorio usadas. Esta información es de alta relevancia para poder crear normas adecuadas a nuestra realidad.

Efectuamos un estudio piloto prospectivo el año 2009, que incluyó a 100 embarazadas iniciando control prenatal en el Hospital Clínico de la Pontificia Universidad Católica de Chile, utilizando como valores de normalidad los sugeridos por Endocrine Society ${ }^{4}$; detectamos 2\% de hipotiroidismo, 19\% de hipotiroidismo subclínico y $1 \%$ de hipertiroidismo subclínico ${ }^{9}$; frecuencias que resultaron muy superiores a las publicadas en otras poblaciones.

Considerando la alta frecuencia de hipotiroidismo detectada en embarazadas en nuestro estudio piloto postulamos que la población Chilena embarazada posee una mayor frecuencia de hipotiroidismo que la reportada en otros países. Para poner a prueba nuestra hipótesis, efectuamos un estudio prospectivo con un número mayor de embarazadas chilenas, eligiendo en esta oportunidad una población beneficiaria del sistema público de salud en atención primaria, para evaluar en ellas la incidencia de enfermedad tiroidea funcional y autoinmune.

\section{Materiales y Método}

\section{Pacientes incluidos}

Mujeres embarazadas que iniciaban su control prenatal ambulatorio, en el período de un año (junio de 2010 y julio de 2011), en primer trimestre del embarazo, en los 3 Centros de Salud ANCORA de atención primaria dependientes del Servicio de Salud Metropolitano Sur-Oriente en asociación con la Pontificia Universidad Católica de Chile. La población atendida en estos centros es beneficiaria del sistema público y pertenece a las comunas de Puente Alto y La Pintana.

Todas las mujeres que iniciaban su control prenatal en el centro fueron evaluadas como posiblemente incluidas en el estudio, y fueron invitadas a participar una vez que se comprobó que no cumplían con criterios de exclusión. Se definieron los siguientes criterios como exclusión-portadoras de hipo e hipertiroidismo conocido, con o sin tratamiento; usuarias de medicamentos que alteraran la función tiroidea como antidepresivos, anticonvulsivantes y antipsicóticos; embarazadas con antecedentes de cirugía tiroidea o administración de radioyodo; y pacientes con embarazo múltiple. Se incluyó primigestas y multíparas de primer trimestre.

\section{Protocolo}

Este proyecto fue aprobado por el Comité de Ética de Investigación de la Escuela de Medicina de la Pontificia Universidad Católica de Chile y del Servicios Metropolitano de Salud Sur Oriente; las participantes fueron incluidas posterior a la firma del Consentimiento Informado. La edad gestacional fue determinada mediante fecha de última menstruación y confirmada con ultrasonografía. Se creó una ficha clínica estandarizada desde donde se obtuvo los antecedentes demográficos y obstétricos de cada participante; especial énfasis en irregularidad menstrual, restricción del creci- 
miento intrauterino (RCIU), abortos espontáneos, parto prematuro, preeclampsia, hijos con retraso del desarrollo psicomotor; además de antecedentes médicos personales como bocio, radioterapia cervical, antecedentes familiares en $1^{\circ}$ ó $2^{\circ}$ grado de enfermedad tiroidea u otra patología autoinmune.

A cada embarazada se le tomó una muestra de $10 \mathrm{ml}$ de sangre; las muestras fueron trasladadas a $4^{\circ} \mathrm{C}$ y procesadas por el Laboratorio Clínico de la Pontificia Universidad Católica de Chile. Se obtuvo para cada embarazada, niveles de TSH, T4 total, T4 libre (T4L) y anticuerpos antiTPO.

\section{Mediciones de laboratorio}

TSH ultrasensible, T4 total y T4L fueron medidas por inmunoensayo competitivo de electroquimioluminiscencia (Modular Analitics E 170, ROCHE). Para TSH, la detección mínima fue $0,005 \mathrm{uUI} / \mathrm{mL}$ y el coeficiente de variación fue $7,2 \%$ para una concentración de $0,035 \mu \mathrm{UI} /$ mL y 3,3\% para una concentración de 3,66 $\mu \mathrm{UI} /$ $\mathrm{mL}$ durante el período del estudio. En el caso de T4 total, la sensibilidad analítica fue $0,42 \mu \mathrm{g} /$ $\mathrm{dL}$ con un coeficiente de variación de $3,7 \%$ para niveles de $5,1 \mu \mathrm{g} / \mathrm{dL}$ y $4,2 \%$ para niveles de $18 \mu \mathrm{g} /$ dL. Para T4 libre (T4L), la sensibilidad analítica fue $0,023 \mathrm{ng} / \mathrm{dL}$ con coeficiente de variación $2,7 \%$ para concentración de $1,16 \mathrm{ng} / \mathrm{dL}$ y de $3,6 \%$ para concentración de 2,0 ng/ dL.

La medición de anticuerpos antiTPO se efectuó con determinación cuantitativa de anticuerpos antiperoxidasa tiroidea (antiTPO), a través de un enzimoinmunoensayo de micropartículas (AXSYM-ABBOT) con una sensibilidad analítica de $1 \mathrm{UI} / \mathrm{mL}$.

\section{Definiciones}

Se utilizó como patrón de referencia de normalidad, el nivel de hormonas tiroideas normales ajustadas para cada trimestre de embarazo según guías internacionales ${ }^{4}$. Todas las muestras correspondieron a primer trimestre de embarazo, de modo que se utilizó como rango normal los siguientes: TSH $[0,03-2,50 \mathrm{uUI} / \mathrm{mL}] ; \mathrm{T} 4$ total [6,9-18 ug/dL] (1,5 veces el rango de normalidad de laboratorio en no embarazadas); T4 libre [0,93$1,70 \mathrm{ng} / \mathrm{dL}]$ y antiTPO $[<12 \mathrm{UI} / \mathrm{mL}]$.

Se definió como hipotiroidismo a las embarazadas con TSH > $10 \mathrm{uUI} / \mathrm{mL}$ y T4 total o T4L bajas; hipotiroidismo subclínico con TSH 2,51-10 uUI/ $\mathrm{mL}$, asociadas a T4 total y T4L dentro de límites normales; hipertiroidismo subclínico con TSH $<0,03 \mathrm{uUI} / \mathrm{mL}$, asociada a T4 total y T4L normal.

Se definió como factor de riesgo de enfermedad tiroidea aquellos descritos en las guias de manejo de disfunción tiroidea publicadas por la Endocrine Society en año 2007, vigentes al momento del inicio del estudio entre las que se incluyen las descritas en la Tabla $3^{4}$.

\section{Tamaño muestral}

Se calculó el tamaño muestral para un estudio de prevalencia, con una precisión de 95\% (5\% de error), asumiendo una estimación de prevalencia de $10 \%$ (prevalencia reportada internacionalmente), con un nivel de confianza de 95\% (5\% de error alfa), obteniéndose un tamaño muestral de 477 mujeres. Considerando el número histórico de ingresos a control prenatal en los tres Centros de Salud ANCORA (1.500-1.600 por año), y que probablemente $60-70 \%$ lo hacen en el primer trimestre, planeamos que se lograría el tamaño muestral en un año. Decidimos incluir a todas las embarazadas que cumplieran con los criterios de inclusión en un año.

\section{Estadística}

Todos los sets de datos fueron sometidos a un test de normalidad (Kolmogorov-Smirnov test), y se compararon luego con test paramétricos o no paramétricos según fuera apropiado. La comparación entre la frecuencia de factores de riesgo se efectuó mediante la prueba de chi-cuadrado $\left(\chi^{2}\right)$ con corrección de Yates. El análisis de riesgo se realiza según riesgo relativo (RR) con intervalo de confianza de 95\% (IC95\%). Se consideró diferencia significativa un $\mathrm{p}<0,05$.

\section{Resultados}

Entre junio de 2010 y julio de 2011, ingresaron a control prenatal 1.565 embarazadas; 994 cursando primer trimestre. De ellas, se analizaron para ser incluidas en el estudio 865 embarazadas (87\% de las posibles); $23 \%$ fueron excluidas de acuerdo a los criterios mencionados ( $11 \%$ por tener antecedentes de alguna patología tiroidea previa y $12 \%$ por las otras causas), 5\% rechazaron participar, $13 \%$ fueron pérdidas por otras causas (se fueron a otro centro, no se pudo obtener las muestras de sangre o información clínica incompleta o no 
analizable). Finalmente, fueron estudiadas 510 embarazadas, en todas ellas se obtuvo las muestras y la historia clínica necesarias para el análisis (Figura 1).

En la Tabla 1, se aprecian las características clínicas de las embarazadas participantes cuya edad promedio fue 25,7 $( \pm 6,6)$ años con edad gestacional al ingreso de $8,8( \pm 2,5)$ semanas; hubo $40,4 \%$ de primíparas y el IMC promedio fue 26,1 $( \pm 5,0) \mathrm{kg} / \mathrm{m}^{2}$.

En la Tabla 2 se muestran los valores mediana

Tabla 1. Características de la población de embarazadas incluidas en el estudio

\begin{tabular}{|c|c|}
\hline \multicolumn{2}{|l|}{ Características } \\
\hline Edad materna promedio, años \pm DS & $25,7 \pm 6,6$ \\
\hline $\begin{array}{l}\text { Edad materna, n (\%) } \\
\text { Menor a } 18 \\
18-24 \\
25-34 \\
35 \text { y más }\end{array}$ & $\begin{array}{l}37(7,3) \\
217(42,5) \\
196(38,4) \\
60(11,8)\end{array}$ \\
\hline Edad gestacional al ingreso, sem ( \pm DS) & $8,8( \pm 2,5)$ \\
\hline $\begin{array}{l}\text { Edad gestacional, } n(\%) \\
<9 \text { sem } \\
9 a<11 \text { sem } \\
11 a<15 \text { sem }\end{array}$ & $\begin{array}{l}283(55,5) \\
113(22,2) \\
114(22,3)\end{array}$ \\
\hline $\begin{array}{l}\text { Historia de embarazos previos, n (\%) } \\
\text { Ninguno } \\
\text { Un embarazo } \\
\text { Dos embarazos } \\
\text { Más de dos embarazos }\end{array}$ & $\begin{array}{l}206(40,4) \\
157(30,8) \\
104(20,4) \\
43(8,4)\end{array}$ \\
\hline $\begin{array}{l}\mathrm{IMC} \text { promedio } \mathrm{kg} / \mathrm{m}^{2} \pm \mathrm{DS} \\
\mathrm{IMC} \text { rango }\end{array}$ & $\begin{array}{l}26,1 \pm 5.0 \\
16,3-42,1\end{array}$ \\
\hline $\begin{array}{l}\text { Estado nutricional al ingreso, n (\%) } \\
\text { Bajo peso (IMC < 18,5) } \\
\text { Normo peso (IMC 18,5-24,9) } \\
\text { Sobrepeso (IMC 25,0-29,9) } \\
\text { Obesidad (IMC > 30,0) }\end{array}$ & $\begin{array}{l}7(1,4) \\
240(47,1) \\
156(30,6) \\
107(21,0)\end{array}$ \\
\hline
\end{tabular}

y percentiles 2,5 y 97,5 de los exámenes funcinales tiroideos realizados. Se puede observar que el valor de mediana de TSH es levemente inferior si se toman sólo las pacientes sin presencia de anticuerpos antitiroideos lo mismo ocurre con el percentil 97,5 de TSH, en los valores de T4 total y libre no existen estas diferencias.

En la Figura 2, se muestra el porcentaje de embarazadas con patología tiroidea funcional detectada. Cero coma seis por ciento (3 participantes) corresponde a hipotiroidismo clínico; 35,3\%

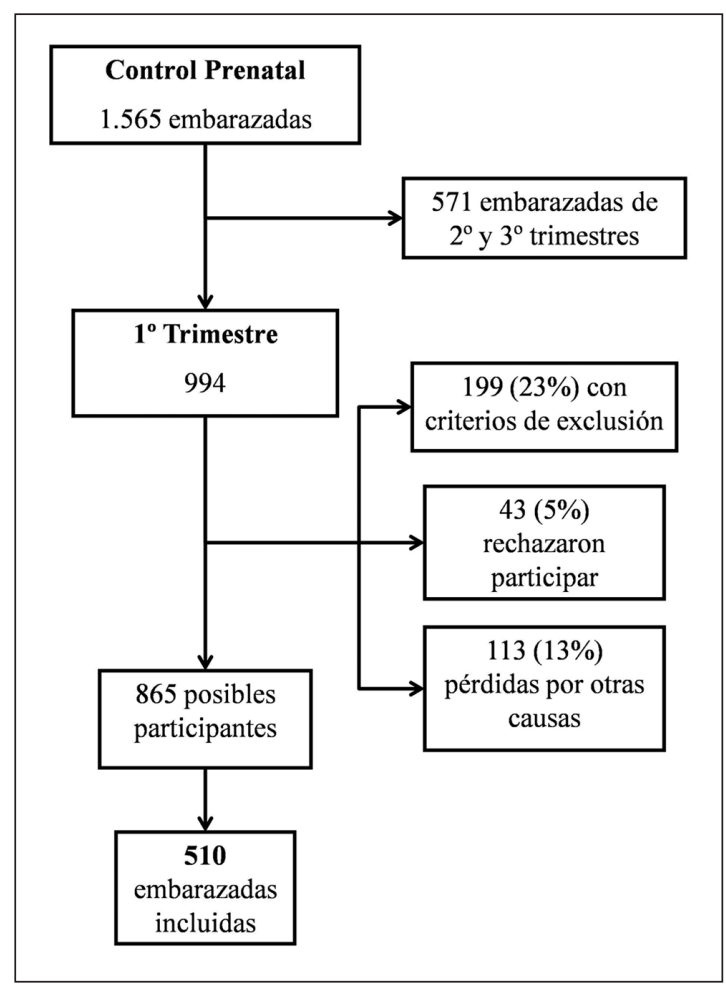

Figura 1. Diagrama de flujo de las embarazadas incluidas en el estudio.

Tabla 2. Resultados exámenes de laboratorio realizados en población total de pacientes en primer trimestre embarazo y en pacientes sin la presencia de anticuerpos anti TPO

\begin{tabular}{|lcccccc|}
\hline & \multicolumn{3}{c}{$\mathbf{n = 5 1 0}$ total pacientes } & \multicolumn{3}{c|}{$\mathbf{n = 4 6 1}$ pacientes sin Anticuerpos TPO } \\
& TSH & T4 Total & T4 libre & TSH & T4 total & T4 libre \\
Mediana & 2,0300 & 9,5000 & 1,1300 & 1,9600 & 9,5000 & 1,1300 \\
Percentil 2,5 &, 1078 & 6,6000 &, 8278 &, 0955 & 6,7000 &, 8555 \\
Percentil 97,5 & 6,5568 & 14,2225 & 1,4723 & 5,4590 & 14,3000 & 1,4935 \\
\hline
\end{tabular}




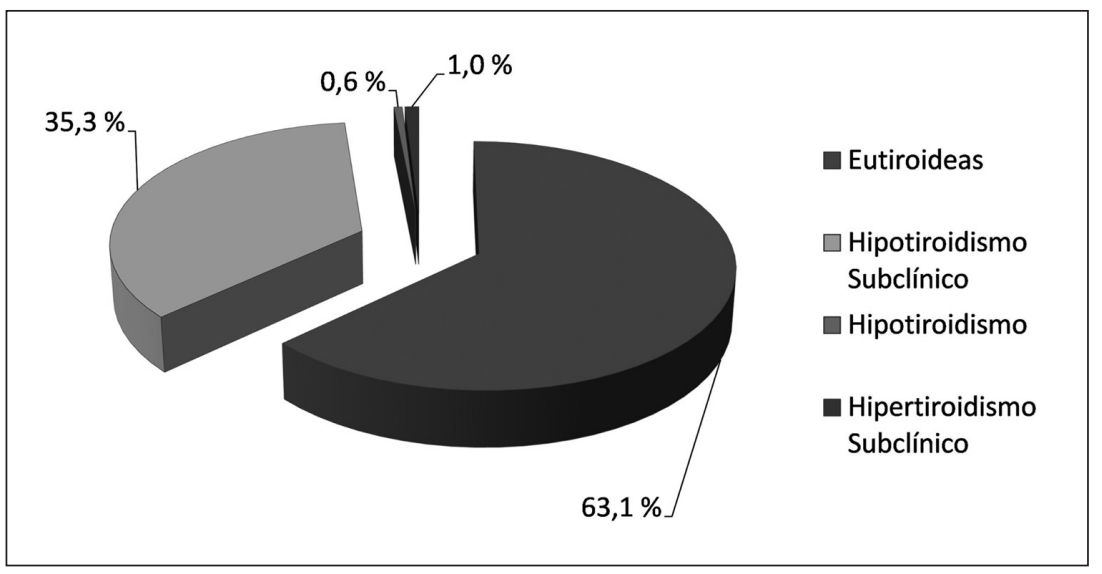

Figura 2. Frecuencia de patología tiroidea funcional en embarazadas chilenas en control prenatal en atención primaria pública del área suroriente de Santiago.
(180 participantes) hipotiroidismo subclínico; y $1 \%$ (5 participantes) hipertiroidismo subclínico. $63,1 \%$ (322 participantes) de las embarazadas no presentó patología funcional.

Nueve coma cuatro por ciento (48 participan-

Tabla 3. Distribución de las embarazadas portadoras de anticuerpos anti-tiroperoxidasa (antiTPO)

\begin{tabular}{|lrc|}
\hline & AntiTPO positivo, n (\%) \\
\hline Hipotiroidismo & 2 & $(4,2)$ \\
Hipotiroidismo subclínico & 28 & $(58,3)$ \\
Hipertiroidismo subclínico & 0 & $(0)$ \\
Eutiroideas & 18 & $(37,5)$ \\
Total & $48(100)$ \\
\hline
\end{tabular}

tes) tuvo anticuerpos antiTPO positivos. Dos de ellas en el grupo de hipotiroidismo clínico, 28 en el grupo con hipotiroidismo subclínico y 18 en la población eutiroidea (Tabla 3 ).

Con respecto a la presencia de antecedentes médicos y factores de riesgo de enfermedad tiroidea asociados a la patología tiroidea materna pesquisada al ingresar al estudio, ninguna de las variables mostró una asociación significativa. (Tabla 4).

\section{Discusión}

Reportamos una elevada frecuencia de patología tiroidea funcional $(36,9 \%)$ en esta población general de embarazadas chilenas cursando primer trimestre de embarazo al utilizar como parámetro el punto de corte de TSH propuesto en las guías

Tabla 4. Factores de riesgo de enfermedad tiroidea en la población incluida. Ninguno de los factores de riesgo se asoció a un elevado riesgo de alteración tiroidea funcional

\begin{tabular}{|c|c|c|c|c|}
\hline & $\begin{array}{l}\text { Sanas } \\
\text { n } \quad(\%)\end{array}$ & $\begin{array}{c}\text { Alteración funcional } \\
n \quad(\%)\end{array}$ & Valor $\mathbf{p}$ & RR (IC 95\%) \\
\hline Infertilidad & $30 \quad(9,4 \%)$ & $21(11,2 \%)$ & 0,62 & $0,84[0,49-1,42]$ \\
\hline Aborto espontáneo & $49(15,4 \%)$ & $36(19,1 \%)$ & 0,33 & $0,80[0,54-1,19]$ \\
\hline Parto < 37 sem & $29(9,1 \%)$ & $13(6,9 \%)$ & 0,49 & $1,31[0,70-2,47]$ \\
\hline Bocio & $2 \quad(0,6 \%)$ & $2(1,1 \%)$ & 0,99 & $0,59[0,08-4,15]$ \\
\hline RT cervical & $0 \quad(0,0 \%)$ & $1 \quad(0,5 \%)$ & 0,79 & nc \\
\hline Diabetes & $3(0,9 \%)$ & $4 \quad(2,1 \%)$ & 0,48 & $0,44[0,10-1,95]$ \\
\hline Hist. familiar & $58(18,2 \%)$ & $32(17,0 \%)$ & 0,83 & $1,07[0,72-1,58]$ \\
\hline Uno o más FR & $123(38,6 \%)$ & $77(41,0 \%)$ & 0,66 & $0,94[0,75-1,17]$ \\
\hline
\end{tabular}

$\mathrm{RT}=$ radioterapia; Hist. Familiar $=$ historia de familiares de $1^{\circ}$ ó $2^{\circ}$ grado con patología tiroidea; $F R=$ factores de riesgo. 
internacionales. No observamos una correlación entre la presencia de factores de riesgo de enfermedad tiroidea definidos por recomendaciones de las guías internacionales ${ }^{4}$ y el hallazgo de patología tiroidea funcional ni autoinmune.

La alta frecuencia de hipotiroidismo que encontramos contrasta con reportes internacionales, en que se ha determinado alrededor de 1 a $2 \%$ de hipotiroidismo y 4-8\% de hipotiroidismo subclínico en gestantes provenientes de áreas suficientes de yodo ${ }^{10}$. La frecuencia de hipotiroidismo que reportamos $(0,6 \%)$ es similar a la descrita, pero la prevalencia de hipotiroidismo subclínico es significativamente mayor (35,3\%). Esta diferencia no parece explicable por un déficit de yodo, dado que en Chile existe yodación de sal desde el año 1979. Sin embargo, para descartar completamente esta posibilidad, debe medirse niveles de yoduria, un análisis no efectuado en esta investigación.

Una explicación razonable para la alta frecuencia de hipotiroidismo subclínico es que se deba al uso de puntos de corte inapropiado para nuestra población. Esta interrogante es similar a la que enfrentamos hoy en la población general adulta no embarazada, donde la reciente Encuesta Nacional de Salud (ENS 2009-10) mostró también alta prevalencia de hipotiroidismo usando los puntos de corte de poblaciones extranjeras ${ }^{11}$.

Hay que considerar que en las publicaciones realizadas hasta ahora existe una variabilidad importante en los niveles de TSH para poder definir normalidad en las gestantes. Especialmente variable es el corte superior de TSH, el que podría estar influido por múltiples factores como: la etnia de origen, la edad media de las gestantes, diferentes métodos de análisis, el estatus de iodo, método de cálculo y las diversas consideraciones para la selección de pacientes "sanas"12. En nuestros datos, al excluir a la población con TPO positivo, obtuvimos un punto de corte de TSH en 5,4, muy superior al propuesto en las guías internacionales y sólo semejante al encontrado en una población en India ${ }^{13}$.

En cuanto al hipertiroidismo clínico, la literatura consigna una prevalencia de $0,2 \%$ de las gestantes y el hipertiroidismo subclínico se encuentra en $0,4 \%$ de los embarazos ${ }^{14}$; de ellas la mayoría correspondería a hipertiroidismo gestacional, que no se asociaría a eventos adversos maternos ni fetales, por lo tanto, un valor de TSH bajo el límite de detección, con hormonas periféricas normales, habitualmente no sería significativo clínicamen$t^{15}$. En nuestro caso, no detectamos embarazadas con hipertiroidismo clínico pero tuvimos $1 \%$ de hipertiroidismo subclínico, que es semejante a lo expuesto internacionalmente.

La literatura describe que la enfermedad tiroidea autoinmune, con o sin disfunción tiroidea, se observa en 8 a 15\% de las embarazadas ${ }^{16,17}$. En nuestro caso, la presencia de anticuerpos antiTPO positivos $(9,4 \%)$ se encontró dentro del rango descrito, demostrando que nuestra población es similar a otras y descartando fenómenos de autoinmunidad como la principal causa de la alta incidencia de hipotiroidismo subclínico.

En relación a la asociación entre factores de riesgo de enfermedad tiroidea y patología tiroidea funcional y autoinmune pesquisada durante la gestación, no observamos una asociación estadísticamente significativa, sugiriendo que el tamizaje sólo en base a estos factores de riesgo dejaría sin diagnóstico a un alto porcentaje de embarazadas. En el caso de nuestro estudio, 59\% de las embarazadas con alteraciones tiroideas funcionales no tenían factores de riesgo.

Otros grupos, al igual que lo que aquí reportamos, han sugerido que realizar test de función tiroidea sólo dirigido a embarazadas con factores de riesgo clínico, no detectó entre 30 y $80 \%$ de las gestantes con disfunción tiroidea, al compararlas con el tamizaje universal ${ }^{18-21}$.

Sin embargo, la recomendación de la Endocrine Society ${ }^{4}$ es el tamizaje de las gestantes con alto riesgo de enfermedad tiroidea, incluyendo a embarazadas con historia personal o familiar de patología tiroidea; historia personal de DM tipo $1 \mathrm{u}$ otra enfermedad autoinmune; clínica sugerente de disfunción tiroidea; bocio; anticuerpos antitiroideos positivos; antecedentes de radiación cervical, aborto, parto prematuro o infertilidad.

El argumento principal en contra del tamizaje universal ha sido la falta de estudios que demuestren costo-efectividad de esta conducta, específicamente el beneficio de la detección y tratamiento de mujeres con hipotiroidismo subclínico. Existe hasta ahora un solo trabajo que no demostró beneficio de la terapia con levotiroxina en el desarrollo intelectual (seguimiento a tres años) de los hijos de embarazadas portadoras de hipotiroidismo subclínico ${ }^{22}$.

En resumen, observamos una elevada frecuencia de hipotiroidismo subclínico en la población 
estudiada, sugiriendo una alta incidencia de esta patología tiroidea funcional en la población general de embarazadas en Chile. Si bien no tenemos una explicación para este fenómeno, consideramos que debe ser investigado adicionalmente para mejorar las estrategias de detección y tratamiento en nuestro país. Es evidente que los antecedentes de la historia clínica no permiten identificar a aquella población con alteraciones funcionales tiroideas. En este escenario, el tamizaje universal parece más razonable, especialmente si se considera la alta frecuencia de alteraciones detectadas. La decisión de efectuar tamizaje universal en nuestra población, debe debatirse desde el punto de vista de salud pública, y debe necesariamente ser precedida de un dato más universal de puntos de corte nacional ya que implicaría un costo cuya efectividad aun no está bien establecida.

\section{Referencias}

1. Glinoer D. The regulation of thyroid function in pregnancy: pathways of endocrine adaptation from physiology to pathology. Endocrine reviews 1997; 18 (3): 404-33.

2. Mandel S, Spencer C. Are detection and treatment of thyroid insufficiency in pregnancy feasible? Thyroid 2005; 15 (1): 44-53.

3. Abalovich M, Gutiérrez S, Alcaraz G, Maccallini G, Garcia A, et al. Overt and subclinical hypothyroidism complicating pregnancy. Thyroid 2002; 12 (1): 63-8.

4. Abalovich M, Amino N, Barbour LA, Cobin RH, De Groot LJ, Glinoer D, et al. Management of Thyroid Dysfunction during Pregnancy and Postpartum: An Endocrine Society Clinical Practice Guideline. Journal of Clinical Endocrinology \& Metabolism 2007; 92 (8): s1-47.

5. Stagnaro-Green A, Abalovich M, Alexander E, Azizi F, Mestman J, Negro R, et al. Guidelines of the American Thyroid Association for the Diagnosis and Management of Thyroid Disease During Pregnancy and Postpartum. Thyroid 2011; 21 (10): 1-45.

6. Berbel P, Mestre JL, Santamaría A, Palazón I, Franco A, Graells M, et al. Delayed neurobehavioral development in children born to pregnant women with mild hypothyroxinemia during the first month of gestation: the importance of early iodine supplementation. Thyroid 2009; 19 (5): 511-9.

7. Vanderpump MPJ, Tunbridge WMG. Epidemiology and prevention of clinical and subclinical hypothyroidism. Thyroid 2002; 12 (10): 839-47.
8. Pop VJ, Brouwers EP, Vader HL, Vulsma T, van Baar AL, de Vijlder JJ. Maternal hypothyroxinaemia during early pregnancy and subsequent child development: a 3-year follow-up study. Clinical endocrinology 2003; 59 (3): 282-8.

9. Castillo C, García M, Martínez C, Carvajal J, Campino $\mathrm{C}$, Arteaga $\mathrm{E}$ et al. Alta frecuencia de patología tiroidea funcional y autoinmune en mujeres embarazadas chilenas catalogadas como sanas. Endocrinología y Diabetes 2009; 2 (3): 147-53.

10. Lazarus JH. Screening for thyroid dysfunction in pregnancy: is it worthwhile? Journal of thyroid research 2011; 2011: 397012. Available from: http://www.pubmedcentral.nih.gov/articlerender.fcgi?artid=3134289\&tool $=$ pmcentrez\&rendertype $=$ abstract

11. Encuesta nacional de salud. Chile 2009-2010 1. 2010; Available from: http://www.encuestasalud.cl/ens/wpcontent/uploads/2011/09/InformeENS_2009-2010_ CAP5.pdf

12. Yan Y-Q, Dong Z-L, Dong L, Wang F-R, Yang X-M, Jin $\mathrm{X}-\mathrm{Y}$, et al. Trimester -and method- specific reference intervals for thyroid tests in pregnant Chinese women: methodology, euthyroid definition and iodine status can influence the setting of reference intervals. Clinical Endocrinology 2011; 74 (2): 262-9.

13. Marwaha RK, Chopra S, Gopalakrishnan S, Sharma B, Kanwar RS, Sastry A, et al. Establishment of reference range for thyroid hormones in normal pregnant Indian women. BJOG 2008; 115: 602-6.

14. Mestman JH. Hyperthyroidism in pregnancy. Endocrinology and metabolism clinics of North America 1998; 27 (1): 127-49.

15. Casey BM, Dashe JS, Wells CE, McIntire DD, Leveno KJ, Cunningham FG. Subclinical hyperthyroidism and pregnancy outcomes. Obstetrics and Gynecology 2006; 107: 337-41.

16. Stagnaro-Green A, Roman SH, Cobin RH, El-Harazy E, Álvarez-Marfany M, Davies TF. Detection of atrisk pregnancy by means of highly sensitive assays for thyroid autoantibodies. JAMA 1990; 264 (11): 1422-5.

17. Lee RH, Spencer CA, Montoro MN, Aghajanian P, Goodwin TM, Miller EA, et al. Effect of thyroid peroxidase antibodies on thyroid-stimulating hormone reference limits in a primarily Latina population. Obstetric Medicine 2009; 2 (4): 154-6.

18. Lazarus JH, Premawardhana LDKE. Screening for thyroid disease in pregnancy. Journal of clinical pathology 2005; 58 (5): 449-52.

19. Negro R, Schwartz A, Gismondi R, Tinelli A, Mangieri $\mathrm{T}$, Stagnaro-Green A. Universal screening versus case finding for detection and treatment of thyroid hormonal 
dysfunction during pregnancy. The Journal of clinical endocrinology and metabolism 2010; 95 (4): 1699-707.

20. Chang DLF, Leung AM, Braverman LE, Pearce EN. Thyroid testing during pregnancy at an academic Boston Area Medical Center. The Journal of Clinical Endocrinology and Metabolism 2011; 96 (9): E1452-6.

21. Vaidya B, Anthony S, Bilous M, Shields B, Drury J, Hut- chison S, et al. Detection of thyroid dysfunction in early pregnancy: Universal screening or targeted high-risk case finding? The Journal of clinical endocrinology and metabolism 2007; 92 (1): 203-7.

22. Lazarus J. Pregnancy and thyroid diseases. Presented at the 14th International Thyroid Congress, Paris, France, 2010 (Abstract SY18). 